\title{
SHARP BOUNDARY TRACE INEQUALITIES
}

\author{
GILES AUCHMUTY
}

\begin{abstract}
This paper describes sharp inequalities for the trace of Sobolev functions on the boundary of a bounded region $\Omega \subset \mathbb{R}^{N}$. The inequalities bound (semi-)norms of the boundary trace by certain norms of the function and its gradient on the region and two specific constants $k_{\rho}$ and $k_{\Omega}$ associated with the domain and a weight function. These inequalities are sharp in that there are functions for which equality holds. Explicit inequalities in some special cases when the region is a ball, or the region between two balls, are evaluated.
\end{abstract}

\section{INTRODUCTION}

This paper describes some elementary, but sharp, trace inequalities. It will be proved that if $\rho$ is a positive weight function on the boundary $\partial \Omega$ of a bounded region $\Omega$, then there are positive constants $k_{\rho}, k_{\Omega}$ such that

$$
\int_{\partial \Omega} \rho|\gamma(u)| d \sigma \leq k_{\rho} \int_{\Omega}|u| d x+k_{\Omega} \int_{\Omega}|\nabla u| d x
$$

for all $u \in W^{1,1}(\Omega)$. Here $\gamma$ is the boundary trace operator and mild conditions on the boundary $\partial \Omega$ and the function $\rho$ are required. $k_{\rho}, k_{\Omega}$ are characterized explicitly and equality holds here when $u \equiv c$ on $\bar{\Omega}$.

Moreover, when $r>1$, inequalities of the form

$$
\int_{\partial \Omega} \rho|\gamma(u)|^{p} d \sigma \leq k_{\rho} \int_{\Omega}|u|^{p} d x+p k_{\Omega}\|u\|_{q}^{p-1}\|\nabla u\|_{r}
$$

hold for $u \in W^{1, r}(\Omega)$, certain ranges of $p$ (depending only on $r$ ) and for a specific value of $q$ that depends on $r$ and $p$. See theorems 5.1 and 6.3 below. The constants $k_{\rho}$ and $k_{\Omega}$ are the same in these two inequalities and may be regarded as geometrical quantities associated with the region $\Omega$ and the weight function $\rho$. In particular, $k_{\Omega}$ is the $L^{\infty}$ norm of the gradient of a function $w_{\rho}$ characterized by the region and $\rho$. This function will be called the trace weight function and is the solution of a variational problem for the torsion equation.

When $\rho \equiv 1$ on $\partial \Omega$ these inequalities can be used to estimate the norms of the trace operator $\gamma: W^{1, r}(\Omega) \rightarrow L^{p}(\partial \Omega, d \sigma)$. Taking $\rho$ to be the characteristic function of a proper subset of the boundary leads to restricted trace estimates.

Date: October 5, 2012.

Key words and phrases. Boundary trace inequalities, Torsion equation, Trace theorem.

This research was partially supported by NSF award DMS 11008754. 
The inequalities described here are much simpler than the trace results described in texts such as those of DiBenedetto [5], chapter IX.18 or Leoni [9], chapter 15. Grisvard [8], theorem 1.5.1.10 describes a family of trace inequalities that follow from the case where $p=q=r>1$ in (1.2) above.

A number of papers have treated issues regarding the "best" constant for various Sobolev trace inequalities - and whether there are Sobolev functions that attain these optimal values. That is, they have proved results about the norm of the trace operator either as a map of $W^{1,1}(\Omega)$ to $L^{1}(\partial \Omega, d \sigma)$ or from $W^{1, p}(\Omega)$ to various $L^{q}(\partial \Omega, d \sigma)$. See Andreu, Mazon, Rossi [1], Border, Rossi, Ferreira [3], Motron [11] for example. These inequalities do not have the scaling properties, or geometrical interpretations, of (1.1) or (1.2) and assume $\rho \equiv 1$. Inequalities such as some of those treated in Auchmuty [2] or (2) of Maggi and Vilani [10] are quite different to the ones to be investigated here - even though they involve the same functionals.

\section{Definitions and Notation}

A region is a non-empty open connected set in $\mathbb{R}^{N}$ with $N \geq 2$. Let $L^{p}(\Omega), p \in[1, \infty]$ be the usual real Lebesgue spaces of functions on $\Omega$. All functions here take values in $\overline{\mathbb{R}}:=[-\infty, \infty]$ and the norm on $L^{p}(\Omega)$ is denoted $\|\cdot\|_{p}$. A function is said to be positive on a set $\mathrm{E}$ if $f(x) \geq 0$ for all $x$ in $\mathrm{E}$. It is strictly positive if $f(x)>0$ for all $x$ in $\mathrm{E}$. When $\Omega$ has finite Lebesgue measure, then $\bar{u}:=|\Omega|^{-1} \int_{\Omega} u d x$ will be the mean value of $u$ on $\Omega$.

For $1 \leq p<\infty, W^{1, p}(\Omega)$ is the Sobolev space of Lebesgue measurable functions on $\Omega$ that are $L^{p}$ and whose weak derivatives $D_{j} u$ are again in $L^{p}$ for each $j \in I_{N}:=\{1,2, \ldots, N\}$. $W^{1, p}(\Omega)$ is a real Banach space with respect to the norm defined by

$$
\|u\|_{1, p}^{p}:=\int_{\Omega}\left[|u(x)|^{p}+|\nabla u(x)|^{p}\right] d x .
$$

Here $\nabla u:=\left(D_{1} u, D_{2} u, \ldots, D_{N} u\right)$ is the gradient of the function $u$ and $|v|$ will denote the 2-norm of a vector $\mathrm{v}$.

The definitions and terminology of Evans and Gariepy [6], will generally be followed except that $\sigma, d \sigma$, respectively, represent Hausdorff $(N-1)$-dimensional measure and integration with respect to this measure. Also Hausdorff $(N-1)$-dimensional measure will be called surface area measure in this paper. In particular we require that a unique unit outward normal function $\nu: \partial \Omega \rightarrow S_{1}$ be defined $\sigma$ a.e. on $\partial \Omega$. Here $S_{1}$ is the unit sphere in $\mathbb{R}^{N}$.

$H^{1}(\Omega)$ will denote the standard Sobolev space of Lebesgue measurable functions on $\Omega$ that are $L^{2}$ and whose weak derivatives $D_{j} u$ are again in $L^{2}$ for each $j \in I_{N}:=\{1,2, \ldots, N\}$. $H^{1}(\Omega)$ is a real Hilbert space under the standard $H^{1}-$ inner product

$$
[u, v]_{1,2}:=\int_{\Omega}[u(x) v(x)+\nabla u(x) \cdot \nabla v(x)] d x .
$$

and the associated norm is denoted $\|u\|_{1,2}$. 
When $K$ is a compact subset of $\mathbb{R}^{N}$, then $C(K)$ will denote the Banach space of continuous real valued functions on $K$ with the maximum norm. When $\Omega$ is an open set then $C_{b}^{k}(\Omega)$ is the space of uniformly bounded $C^{k}$-functions on $\Omega$.

A bounded region $\Omega$ is said to satisfy the Sobolev imbedding theorem provided the imbedding of $W^{1, p}(\Omega)$ into $L^{q}(\Omega)$ is continuous when $1 \leq p<N$ and $1 \leq q \leq p_{S}$ with $p_{S}=p N /(N-p) . p_{S}$ is called the Sobolev conjugate of $p$. When $p=N$ this imbedding should be continuous for all $1 \leq q<\infty$. These theorems hold under a variety of conditions on the boundary $\partial \Omega$. See [6] or DiBenedetto [5] for statements and proofs of such results.

This paper will describe some inequalities for certain boundary (semi-)norms of Sobolev functions in terms of norms of the functions on $\Omega$. The following condition will be required throughout.

Condition B1: $\Omega$ is a bounded region in $\mathbb{R}^{N}$ and its boundary $\partial \Omega$ is the union of a finite number of disjoint closed Lipschitz surfaces; each surface having finite surface area.

Let $C_{c}^{1}\left(\mathbb{R}^{N}\right)$ be the space of functions that are $C^{1}$ on $\mathbb{R}^{N}$ and have compact support. The Gauss-Green (or divergence) theorem holds on a region $\Omega$ provided for any $v \in C_{c}^{1}\left(\mathbb{R}^{N}\right)$,

$$
\int_{\Omega} D_{j} v d x=\int_{\partial \Omega} v \nu_{j} d \sigma \quad \text { for } j \in I_{N} .
$$

See [6], chapter 5 section 5 , for conditions on the region $\Omega$ and its boundary for which (2.3) holds.

Given a function $v \in C_{c}^{1}\left(\mathbb{R}^{N}\right)$, its restriction to $\bar{\Omega}$ will again be denoted $v$ and the restriction operator is $R_{\Omega}$. The space of all such restrictions will be denoted $C_{R}^{1}(\bar{\Omega})$ and is a subspace of $W^{1, \infty}(\Omega)$. Define $G^{1, p}(\Omega)$ to be the closure of $C_{R}^{1}(\bar{\Omega})$ in the $(1, p)$ norm.

Grisvard [8] theorem 1.4.2.1 quotes a result that $G^{1, p}(\Omega)=W^{1, p}(\Omega)$ when the region $\Omega$ has a "continuous boundary". DiBenedetto [5] propositions 18.1 and 19.1 shows that $G^{1, p}(\Omega)=W^{1, p}(\Omega)$ when the region $\Omega$ satisfies a segment property and provides a counterexample with a disconnected boundary. Many of the standard extension theorems for $C^{1}$ functions on $\Omega$ imply this condition. See the discussion of extension theorems in Brezis [4] for example. Whether or not $W^{1, p}(\Omega)=G^{1, p}(\Omega)$ is a regularity condition on the boundary $\partial \Omega$.

When $u$ is a continuous function on $\bar{\Omega}$, then the boundary trace operator is the linear map $\gamma: C(\bar{\Omega}) \rightarrow C(\partial \Omega)$ that evaluates the function pointwise on the boundary. Our interest here is in the properties of this operator when the domain is a Sobolev space $W^{1, r}(\Omega)$. When $r>N$, this trace operator is well-defined from Morrey's imbedding theorem. For $1 \leq r \leq N, \gamma$ may be extended to be a continuous linear transformation of $W^{1, p}(\Omega)$ into $L^{1}(\partial \Omega, d \sigma)$ provided the boundary satisfies some regularity conditions. See [5], [6] or [8] for descriptions of such results..

The region $\Omega$ is said to satisfy the $W^{1, r}$-trace theorem provided the trace mapping has an extension $\gamma$ that is a continuous linear map of $W^{1, r}(\Omega)$ into $L^{q}(\partial \Omega, d \sigma)$ for all $1 \leq r \leq N$ 
and

$$
1 \leq q \leq p_{T}(r):=\frac{r(N-1)}{N-r} \text { for } r<N, \text { and } 1 \leq q<\infty \text { when } r=N .
$$

The quantity $p_{T}(r)$ is called the critical trace index associated with $r$. Proofs of such results may be found in a number of texts including DiBenedetto [5], section IX.18 and Grisvard [8], section 1.5.

Our basic assumption is that the region $\Omega$ has a boundary $\partial \Omega$ such that

Condition B2: The region $\Omega$ is such that (B1), the Sobolev imbedding and $W^{1, p}$-trace theorems hold and $W^{1, p}(\Omega)=G^{1, p}(\Omega)$.

For regions obeying this condition (B2), the Gauss-Green theorem (2.3) holds for all $v \in W^{1,1}(\Omega)$ with $\gamma v$ replacing $v$ on the right hand side and the derivatives taken in a weak sense; see [6], chapter 5. In the following the trace operator will generally be understood when integrals over $\partial \Omega$ are taken.

\section{The Trace Weight Function}

When (B1) holds, the boundary $\partial \Omega$ is a finite union of compact connected subsets in $\mathbb{R}^{N}$ whose Hausdorff $(N-1)$ dimensional measure is finite and positive. Let $\rho: \partial \Omega \rightarrow[0, \infty]$ be a Borel function that is in $L^{s}(\partial \Omega, d \sigma)$ for some $s \geq 1$ and $M:=\int_{\partial \Omega} \rho d \sigma$.

The sharp inequalities to be derived here involve a function that will be called the trace weight function $w_{\rho}$ of $\rho . w_{\rho}$ is defined as the unique solution of a simple variational principle and may be regarded as a solution of the torsion equation subject to Neumann boundary data. When $\rho \equiv 1$ on $\partial \Omega$ this function is a geometrical quantity associated with the region $\Omega$. Torsion functions, obeying zero Dirichlet boundary conditions, were recently used by Giorgi and Smits [7]. to obtain upper and lower bounds on the principal eigenvalues of Dirichlet-Laplacians on $\Omega$. Their function also has a probabilistic interpretation.

Let $H_{m}^{1}(\Omega)$ be the subspace of $H^{1}(\Omega)$ of all functions that satisfy $\bar{u}=0$. It is a closed subspace of $H^{1}(\Omega)$ and condition (B2) implies that Poincare's inequality holds. Namely there is a $c_{\Omega}>0$ such that

$$
\int_{\Omega}|\nabla u|^{2} d x \geq c_{\Omega} \int_{\Omega}(u-\bar{u})^{2} d x, \text { for all } u \in H^{1}(\Omega) .
$$

Thus $H_{m}^{1}(\Omega)$ is a real Hilbert space with respect to the inner product defined by

$$
\langle u, w\rangle_{\nabla}:=\int_{\Omega} \nabla u \cdot \nabla w d x .
$$

Given $\rho$ as above, define $\mathcal{E}: H_{m}^{1}(\Omega) \rightarrow \mathbb{R}$ by

$$
\mathcal{E}(w):=\int_{\Omega}|\nabla w|^{2} d x-2 \int_{\partial \Omega} \rho w d \sigma .
$$


Consider the variational problem $\left(\mathcal{P}_{\rho}\right)$ of minimizing $\mathcal{E}$ on $H_{m}^{1}(\Omega)$ and evaluating

$$
\alpha(\rho):=\inf _{w \in H_{m}^{1}(\Omega)} \mathcal{E}(w) .
$$

Theorem 3.1. Assume (B2) holds, $N \geq 3$ and $\rho \in L^{s}(\partial \Omega$, $d \sigma)$ with $s \geq s_{c}:=2(1-1 / N)$. Then there is a unique minimizer $w_{\rho}$ of $\mathcal{E}$ on $H_{m}^{1}(\Omega)$. When $N=2$, we require $s>1$. $w_{\rho}$ satisfies

$$
\begin{gathered}
\int_{\Omega} \nabla w \cdot \nabla v d x=\int_{\partial \Omega} \rho v d \sigma \quad \text { for all } v \in H_{m}^{1}(\Omega), \text { and } \\
\alpha(\rho)=-\left\|\nabla w_{\rho}\right\|_{2}^{2}=-\int_{\partial \Omega} \rho w_{\rho} d \sigma .
\end{gathered}
$$

Proof. When the trace theorem holds on $\Omega$ then $w \in H_{m}^{1}(\Omega)$ implies that $w \in L^{p}(\partial \Omega)$ for $p \leq p_{T}(2):=2(N-1) /(N-2)($ and $N \geq 3)$. Thus $\mathcal{E}_{2}(w):=\int_{\partial \Omega} \rho w d \sigma$ will be a continuous linear functional on $H_{m}^{1}(\Omega)$ provided $\rho \in L^{s}(\partial \Omega, d \sigma)$ with $s \geq s_{c}:=2(1-1 / N)$. When $N=2, \mathcal{E}_{2}$ is a continuous linear functional when $s>1$ using the corresponding trace theorem.

Thus our conditions imply that $\mathcal{E}$ is continuous, strictly convex and coercive on $H_{m}^{1}(\Omega)$. Hence there is a unique minimizer of $\mathcal{E}$ on $H_{m}^{1}(\Omega)$. It is easily verified that $\mathcal{E}$ is Gateaux differentiable on $H_{m}^{1}(\Omega)$ with

$$
\langle D \mathcal{E}(w), v\rangle=2 \int_{\Omega} \nabla w \cdot \nabla v d x-2 \int_{\partial \Omega} \rho v d \sigma .
$$

Thus the minimizer of $\mathcal{E}$ on $H_{m}^{1}(\Omega)$ satisfies (3.3).

Put $v=w_{\rho}$ in (3.3) and substitute in the expression (3.2). Then (3.4) follows.

Corollary 3.2. Under the conditions of theorem 3.1, the function $w_{\rho}$ is a weak solution of

$$
\Delta w=k_{\rho}:=M /|\Omega| \text { a.e. on } \Omega \text { and } D_{\nu} w=\rho \quad \text { on } \partial \Omega \text {. }
$$

Proof. Equation (3.3) implies that there is a $c \in \mathbb{R}$ such that

$$
\int_{\Omega} \nabla w \cdot \nabla v d x-\int_{\partial \Omega} \rho v d \sigma=c \int_{\Omega} v d x \text { for all } v \in H^{1}(\Omega)
$$

from a standard orthogonality argument. Take $v \equiv 1$ on $\Omega$ in this equation to see that $c=-M /|\Omega|$. In particular this holds for all $v \in H_{0}^{1}(\Omega)$ and thus $w_{\rho}$ is a weak solution of the equation

$$
-\Delta w=c \quad \text { on } \Omega \text {. }
$$

Using the Gauss-Green theorem and this result we find that

$$
\int_{\partial \Omega} v\left[D_{\nu} w-\rho\right] d \sigma=0 \text { for all } v \in H^{1}(\Omega) .
$$

Since this holds for all $v \in H^{1}(\Omega)$, the boundary condition follows. 
This shows that the function $w_{\rho}$ may be regarded as a weak solution of the torsion equation on $\Omega$ subject to a Neumann boundary condition. Note that $v(x):=w_{\rho}(x)-c|x|^{2}$ is a harmonic function on $\Omega$ when $c=M /(2 N|\Omega|)$ and $v$ obeys

$$
D_{\nu} v=\rho_{1}:=\rho-c(x \cdot \nu) \quad \text { on } \partial \Omega .
$$

Thus $v$, and also $w_{\rho}$, will be $C^{\infty}$ on $\Omega$ as $v$ is harmonic on $\Omega$. For our following analysis the essential condition on the function $\rho$ will be

Condition B3: $\quad \rho \in L^{s}(\partial \Omega, d \sigma)$ with $s \geq s_{c}$ if $N \geq 3$ (or s $>1$ when $\mathrm{N}=2$ ), and $w_{\rho} \in W^{1, \infty}(\Omega)$.

The requirement that $w_{\rho} \in W^{1, \infty}(\Omega)$ holds under various regularity conditions on $\rho$ and $\partial \Omega$. In particular when $\rho \equiv 1$ on $\partial \Omega$, it holds when $\partial \Omega$ satisfies (B2).

\section{Weighted Trace Inequalities.}

In this section some simple sharp trace inequalities will be derived using the divergence theorem and the trace weight function. The inequalities are for the quantity $I_{p}(u):=\int_{\partial \Omega} \rho|\gamma(u)|^{p} d \sigma$ with $p \geq 1$. This integral is the $p$-th power of a (semi-)norm on a class of functions defined on $\partial \Omega$. Our analysis is based on the following result for bounded $C^{1}$ functions on $\Omega$.

Lemma 4.1. Suppose $\Omega$ is a bounded region obeying (B2) and $\rho$ is such that (B3) holds. When $p \in[1, \infty)$,

$$
\int_{\partial \Omega} \rho|\gamma(u)|^{p} d \sigma \leq k_{\rho} \int_{\Omega}|u|^{p} d x+p \quad \int_{\Omega}|u|^{p-1}|\nabla u|\left|\nabla w_{\rho}\right| d x
$$

for all $u \in C(\bar{\Omega}) \cap C_{b}^{1}(\Omega)$.

Proof. Let $w_{\rho}$ be the trace weight function for $\Omega$ as described in the preceding section. The divergence theorem yields

$$
\int_{\Omega} \operatorname{div}\left(|u|^{p} \nabla w_{\rho}\right) d x=\int_{\partial \Omega} \rho|u|^{p} d \sigma
$$

upon using the Neumann condition from (3.5). When $p>1$, this integral also equals

$$
\int_{\Omega}|u|^{p} \Delta w_{\rho} d x+p \int_{\Omega}|u|^{p-2} u \nabla u \cdot \nabla w_{\rho} d x
$$

using a product rule for weak derivatives. Then (4.1) follows from the Poisson equation in (3.5) and elementary inequalities.

When $p=1$, the integral over $\Omega$ becomes

$$
\int_{\Omega}|u| \Delta w_{\rho} d x+\int_{\Omega} \nabla|u| \cdot \nabla w_{\rho} d x
$$


with these derivatives taken in a weak sense. Then (4.1) follows from Corollary 3.2 and the fact that $|\nabla| u||=|\nabla u|$ a.e..

For convenience we shall write $X$ for the space $C(\bar{\Omega}) \cap C_{b}^{1}(\Omega)$. The preceding result extends to $u \in W^{1,1}(\Omega)$ using common Sobolev function approximation arguments. In the following we will use the quantity

$$
k_{\Omega}:=\left\|\left|\nabla w_{\rho}\right|\right\|_{\infty}
$$

It is worth noting that when $\left|\nabla w_{\rho}\right|^{2}$ is continuous on $\bar{\Omega}$, then it is subharmonic on $\Omega$ so it attains its maximum on the boundary $\partial \Omega$. Thus, when $w_{\rho}$ is a classical solution and $\rho$ is continuous on the boundary, then $\sup _{z \in \partial \Omega} \rho(z) \leq k_{\Omega}$ from Corollary 3.2.

Theorem 4.2. Suppose $\Omega$ is a bounded region obeying (B2) and $\rho \in L^{s}(\partial \Omega, d \sigma)$ with $s \geq s_{c}$. Then, for $u \in H^{1}(\Omega)$,

$$
\int_{\partial \Omega} \rho|\gamma(u)| d \sigma \leq k_{\rho} \int_{\Omega}|u| d x+\left\|\nabla w_{\rho}\right\|_{2}\|\nabla u\|_{2}
$$

When (B3) also holds then, for all $u \in W^{1,1}(\Omega)$,

$$
\int_{\partial \Omega} \rho|\gamma(u)| d \sigma \leq k_{\rho} \int_{\Omega}|u| d x+k_{\Omega} \int_{\Omega}|\nabla u| d x
$$

Proof. When $p=1$ the inequalities hold for $u \in X$ from the lemma, properties of the weak derivative and the Cauchy - Schwarz inequality. When $u \in H^{1}(\Omega)$ there is a sequence $\left\{u_{m}\right\} \subset X$ that converges to $u$ in $H^{1}(\Omega)$. The trace operator $\gamma$ is continuous for the region $\Omega$ as (B2) holds, so $\gamma\left(u_{m}\right)$ converges to $\gamma(u)$ in $L^{2}(\partial \Omega, d \sigma)$ and there is a subsequence that converges pointwise to $\gamma(u) \sigma$ a.e. on $\partial \Omega$. Since the inequality (4.3) holds for each $m$, it holds in the limit upon using Fatou's lemma on this subsequence.

Similarly given a $u \in W^{1,1}(\Omega)$, there is a sequence $\left\{u_{m}\right\} \subset X$ that converges to $u$ in $W^{1,1}(\Omega)$. The trace operator $\gamma$ is continuous as a map of $W^{1,1}(\Omega)$ to $L^{1}(\partial \Omega, d \sigma)$ from condition (B2) and (4.4) holds for each $u_{m}$. Fatou's lemma applied to a pointwise convergent subsequence then yields (4.4) for all $u \in W^{1,1}(\Omega)$.

Note that these inequalities are sharp and become equalities when $u \equiv c$ on $\Omega$. When the quantity $k_{\Omega}<\infty$, then $I_{1}(u)$ is finite whenever $u \in W^{1,1}(\Omega)$.

The results show that the boundary integral $I_{1}(u)$ is well-defined and finite whenever $u \in H^{1}(\Omega)$ and $\rho \in L^{s}(\partial \Omega, d \sigma)$ with $s \geq s_{c}$. In particular (4.3) holds without any further regularity assumptions about $\nabla w_{\rho}$. The following sections will describe various sharp $p$-norm analogues of the inequality (4.4). 


\section{Boundary Trace Inequalities When $r>N$.}

Generalizations of the preceding inequality to values $p>1$ depend essentially on proving the continuity of, and finding upper bounds on, the functional $G_{p}$ defined by

$$
G_{p}(u):=\int_{\Omega}|u|^{p-1}|\nabla u| d x .
$$

Note that $G_{p}$ is positive and homogeneous of degree $p$.

Let $X_{p r}$ be the class of all $L^{p}$ functions on $\Omega$ with gradient $|\nabla u| \in L^{r}(\Omega)$. For $p, r \geq 1$, this is a real Banach space with respect to the usual intersection norm;

$$
\|u\|_{p r}:=\|u\|_{p}+\||\nabla u|\|_{r} .
$$

$G_{p}$ is said to be locally bounded on the space $X_{p r}$ if it is bounded on bounded subsets of $X_{p r}$.

Theorem 5.1. Suppose (B2), (B3) hold and $u \in X_{p r}$. If $p>1$ then

$$
\int_{\partial \Omega} \rho|\gamma(u)|^{p} d \sigma \leq k_{\rho} \int_{\Omega}|u|^{p} d x+p k_{\Omega} G_{p}(u)
$$

whenever $G_{p}(u)$ is continuous and locally bounded on $X_{p r}$. If $p \geq p_{0}(r):=2-1 / r$ and $N<r<\infty$, then

$$
\int_{\partial \Omega} \rho|\gamma(u)|^{p} d \sigma \leq k_{\rho} \int_{\Omega}|u|^{p} d x+p k_{\Omega}\|u\|_{q}^{p-1}\|\nabla u\|_{r}
$$

for $u \in X_{p r}$ and $q=\frac{r(p-1)}{r-1} \geq 1$. When $p>1$ and $r=\infty$, then (5.3) holds with $q=p-1$.

Proof. The first inequality holds by an approximation argument. Choose a sequence $\left\{u_{m}\right\} \subset X$ that converges to $u$ in $X_{p r}$ so that the functions converge in $L^{p}$ and their gradients converge to $\nabla u$ in $L^{r}\left(\Omega ; \mathbb{R}^{N}\right)$. Now (5.2) holds for each $u_{m}$. From the proof of lemma 4.1, we have that

$$
\int_{\partial \Omega} \rho\left|u_{m}-u_{k}\right|^{p} d \sigma \leq k_{\rho}\left\|u_{m}-u_{k}\right\|_{p}^{p}+p k_{\Omega} G_{p}\left(u_{m}-u_{k}\right) .
$$

Thus if $G_{p}$ is continuous at 0 , this left hand side is a Cauchy sequence and thus $I_{p}\left(u_{m}\right)$ converges to a unique limit and (5.2) holds by continuity.

Use Holder's inequality in (5.1), to see that

$$
0 \leq G_{p}(u) \leq\|u\|_{q}^{p-1}\|\nabla u\|_{r} \quad \text { with } q=\frac{r(p-1)}{r-1} .
$$

So $G_{p}$ is locally bounded on $X_{p r}$ when $q \geq 1$ or $p \geq 2-1 / r$.

Suppose that the sequence $\left\{u_{m}\right\}$ converges to $\mathrm{u}$ in $X_{p r}$, then

$$
G_{p}(u)-G_{p}\left(u_{m}\right)=\int_{\Omega}|u|^{p-1}\left[|\nabla u|-\left|\nabla u_{m}\right|\right] d x+\int_{\Omega}\left|\nabla u_{m}\right|\left[v-v_{m}\right] d x
$$


where $v=|u|^{p-1}, \quad v_{m}=\left|u_{m}\right|^{p-1}$. Apply Holder's inequality to each of these integrals to obtain

$$
\left|G_{p}(u)-G_{p}\left(u_{m}\right)\right| \leq\|u\|_{q}^{p-1}\left\||\nabla u|-\left|\nabla u_{m}\right|\right\|_{r}+\left\|\left|\nabla u_{m}\right|\right\|_{r}\left\|v-v_{m}\right\|_{r^{\prime}} .
$$

When $r>N$, then Morrey's inequality and the fact that $\Omega$ is a 1-extension region implies that each $u \in X_{p r}$ may be taken to be a continuous bounded function on $\bar{\Omega}$. Then the sequence $\left\{u_{m}\right\}$ converges to $u$ in the supremum norm on $\bar{\Omega}$, so $v_{m}$ also converges to $v$ in this norm and thus $G_{p}\left(u_{m}\right)$ converges to $G_{p}(u)$ from this inequality as $|\Omega|$ is finite. Hence (5.3) follows from the first part of this theorem,.

The last sentence follows from (5.2) upon using Holder's inequality in (5.1).

Note that equality holds in these inequalities for constant functions. When $\rho(z) \equiv 1$ on $\partial \Omega$, these inequalities provide bounds on trace norms of functions in $X_{p r}$ and $W^{1, r}(\Omega)$. This inequality is much simpler than the trace estimates described in the literature such as chapter 9, section 18 of DiBenedetto [5]. In particular it shows that the trace norms depend essentially just on the two geometrical quantities $k_{\rho}$ and $k_{\Omega}$ - and in a simple manner on $p, r$.

When the Sobolev imbedding theorem is invoked this inequality becomes

$$
\int_{\partial \Omega} \rho|u|^{p} d \sigma \leq k_{\rho} \int_{\Omega}|u|^{p} d x+C_{r q} k_{\Omega}\|u\|_{1, r}^{p} \quad \text { for all } \quad u \in W^{1, r}(\Omega)
$$

where $C_{r q}$ depends on the norm of the imbedding of $W^{1, r}(\Omega)$ to $L^{q}(\Omega)$.

When $r>N$ and $p=2-1 / r$, then $q=1$ in (5.3) and $X_{p r}=W^{1, r}(\Omega)$. When $1<$ $p<2-1 / r$, then there is a bound on the boundary 1-seminorm of a function $u \in W^{1, r}(\Omega)$ from the inequality (4.4) and on the (2-1/r)-seminnorm from theorem 5.1. Inequalities for the intermediate p-seminorms may then be found using standard interpolation inequalities.

\section{Boundary Trace Inequalities When $1<r \leq N$.}

Here the preceding inequalities will be extended to situations with $1<r \leq N$. First consider the case where $p=r$, so $X_{p r}=W^{1, p}(\Omega)$.

Theorem 6.1. $\quad$ Suppose (B2), (B3) hold and $p>1$. Then,

$$
\int_{\partial \Omega} \rho|\gamma(u)|^{p} d \sigma \leq k_{\rho} \int_{\Omega}|u|^{p} d x+p k_{\Omega}\|u\|_{p}^{p-1}\|\nabla u\|_{p} \quad \text { for } u \in W^{1, p}(\Omega) .
$$

Proof. In this case the functional $G_{p}$ defined by (5.1) satisfies

$$
0 \leq G_{p}(u) \leq\|u\|_{p}^{p-1}\|\nabla u\|_{p}
$$

upon use of Holder's inequality.

Let $\left\{u_{m}\right\} \subset X$ be a sequence that converges to $u$ in $W^{1, p}(\Omega)$, then the inequality (5.6) holds with $r=p$ and the associated sequence $\left\{v_{m}\right\}$ defined as in the proof of theorem 5.2 is a Cauchy sequence in $L^{p^{\prime}}$. Thus the sequence $I_{p}\left(u_{m}\right)$ is a Cauchy sequence as in (5.4) 
above. Hence $I_{p}(u)$ is well-defined for each $u \in W^{1, p}(\Omega)$ and the fact that (6.1) holds for each $u_{m}$ implies that it also holds for such $u$.

(6.1) is sharp as it becomes an equality for constant functions on $\Omega$. When Young's inequality (with an $\epsilon$ ) is used on the last term here, an inequality of the form given in theorem 1.5.1.10 of Grisvard [8] follows. Grisvard's result involves a general constant $K$ rather than the specific quantities of (6.1). This result is different to the results described in [3] as the expression on the right hand side of (6.1) is not the standard $(1, p)$-norm.

When $r \leq N$ then the boundary trace of a function in $W^{1, r}(\Omega)$ need not be a bounded function on $\partial \Omega$. Suppose $p_{0}(r):=2-1 / r$ and $p_{T}(r)$ is the critical trace index associated with $r$ from (2.4), then there are bounds on $G_{p}$ for a range of values of $p$.

Lemma 6.2. Suppose (B2)-(B3) hold and $r \in(1, N]$ with $N \geq 3$, then $G_{p}$ is locally bounded on $W^{1, r}(\Omega)$ when $p \in\left[p_{0}(r), p_{T}(r)\right]$. When $N=2, r \in(1,2]$, then $G_{p}$ is locally bounded on $W^{1, r}(\Omega)$ for all $p \in\left[p_{0}(r), \infty\right)$. For these values,

$$
0 \leq G_{p}(u) \leq\|u\|_{q}^{p-1}\|\nabla u\|_{r} \quad \text { with } q=\frac{r(p-1)}{r-1} \in\left[1, r_{S}\right]
$$

Proof. This follows from Holder's inequality applied to (5.1) and yields this value of $q$. As $p$ increases from $p_{0}(r)$ to $p_{T}(r)$, the associated values of $q$ increase from 1 to the Sobolev conjugate $r_{S}$.

This enables the following boundary trace inequality.

Theorem 6.3. Suppose (B2), (B3) hold, $r \in(1, N], N \geq 3$ and $p \in\left[p_{0}(r), p_{T}(r)\right]$. Then, for $u \in W^{1, r}(\Omega)$,

$$
\int_{\partial \Omega} \rho|\gamma(u)|^{p} d \sigma \leq k_{\rho} \int_{\Omega}|u|^{p} d x+p k_{\Omega}\|u\|_{q}^{p-1}\|\nabla u\|_{r} .
$$

Here $q=r^{\prime}(p-1) \in\left[1, r_{S}\right]$ with $r^{\prime}$ the conjugate index of $r$. When $N=2, r \in(1,2]$, then this holds for all $p \in\left[p_{0}(r), \infty\right)$.

Proof. To prove this, from theorem 5.1, we need only show that $G_{p}$ is continuous on $W^{1, r}(\Omega)$ since it is locally bounded for this range of $p$ from lemma 6.2. However, just as in $(5.6)$,

$$
\left|G_{p}(u)-G_{p}(w)\right| \leq\|u\|_{q}^{p-1}\||\nabla u|-|\nabla w|\|_{r}+\||\nabla w|\|_{r}\left\||u|^{p-1}-|w|^{p-1}\right\|_{r^{\prime}} .
$$

This implies that $G_{p}$ is continuous when $r^{\prime}(p-1) \geq 1$.

that

When $N \geq 3$ and $r_{S}$ is the Sobolev conjugate of $r$, then there is a constant $C_{r}$ such

$$
\|u\|_{r_{S}} \leq C_{r}\|u\|_{1, r} \text { for all } u \in W^{1, r}(\Omega)
$$

When $q \in\left[p, r_{S}\right]$, the value of $\|u\|_{q}$ can be bounded in terms of the values of $\|u\|_{p}$ and $\|u\|_{r_{S}}$ by the usual interpolation inequalities

$$
\|u\|_{q} \leq\|u\|_{p}^{1-\theta}\|u\|_{r_{S}}^{\theta} \quad \text { with } \quad \theta=\frac{q-p}{r_{S}-p} .
$$


Substituting such bounds into (6.3) one obtains bounds on $I_{p}(u)$ in terms of the $p$-norm of $u$, the $r$-norm of $\nabla u$ and the $(1, \mathrm{r})$ - norm of $u$.

When $r \in(1, N]$ and $p \in(1, r)$, then upper bounds on $I_{p}(u)$ may be found using interpolation between bounds on $I_{1}(u)$ from (4.4) and those of $I_{r}(u)$ from (6.1) as $X_{p r}=$ $W^{1, r}(\Omega)$.

\section{Explicit Trace Inequalities When $\Omega$ is a Ball.}

The preceding inequalities provide some simple bounds when the region $\Omega=B_{R}$ is a ball of radius $R$ in $\mathbb{R}^{N}$ and the function $\rho(z) \equiv 1$ on $\partial \Omega$.

In this case the constants $k_{\rho}=N / R$ and $k_{\Omega}=1$ since the trace weight function is

$$
w_{\rho}(x)=c+\frac{k_{\rho} r^{2}}{2 N}
$$

where $\mathrm{c}$ is a constant. Thus the $W^{1,1}(\Omega)$ trace inequality becomes, from (4.4),

$$
\int_{S_{R}}|u| d \sigma \leq \frac{N}{R} \int_{B_{R}}|u| d x+\int_{B_{R}}|\nabla u| d x .
$$

Here $S_{R}$ is the boundary of $B_{R}$.

For $N \geq 3$, the various cases described above yield that the boundary traces satisfy

$$
\int_{S_{R}}|u|^{p} d \sigma \leq \frac{N}{R}\|u\|_{p}^{p}+p \quad\|u\|_{q}^{p-1}\|\nabla u\|_{r}
$$

for $u \in W^{1, r}(\Omega), q=r^{\prime}(p-1)$ and either (i) $\quad r \in(1, N]$, and $p \in\left[p_{0}(r), p_{T}(r)\right]$, or (ii) $\quad r \in(N, \infty)$ and $p \geq 2-1 / r$, or (iii) $r=\infty, p>1$. Here each of the norms on the right hand side is with respect to the ball $B_{R}$ and equality holds here for constant functions.

When $\Omega:=B_{R} \backslash \bar{B}_{1}$ with $R>1$ is the region between balls of radius $\mathrm{R}$ and radius 1 , then these results yield simple inequalities for both $\int_{S_{1}}|u|^{p} d \sigma$ and $\int_{S_{R}}|u|^{p} d \sigma$ as well as for the integral over the whole boundary. To obtain these inequalities take $\rho$ to be the characteristic function of either the interior boundary $S_{1}$, the exterior boundary $S_{R}$ or the whole boundary respectively. The trace weight functions can be found explicitly in each of these cases and the corresponding values of $k_{\rho}$ will be different, namely:

$$
\frac{N}{R^{N}-1}, \quad \frac{N R^{N-1}}{R^{N}-1} \quad \frac{N\left(R^{N-1}+1\right)}{R^{N}-1}
$$

respectively but $k_{\Omega}=1$ for each of these choices of the boundary integral. 


\section{REFERENCES}

[1] F. Andreu, J.M. Mazou and J.D. Rossi, "The best constant for the Sobolev trace embedding from $W^{1,1}(\Omega)$ into $L^{1}(\partial \Omega, d \sigma)$ ", Nonlinear Analysis, T.M.A. 59 (2004), 1125-1145.

[2] G. Auchmuty, "Optimal coercivity inequalities in $W^{1, p}(\Omega)$, Proc. Royal Soc. Edinburgh, 135A, (2005), 915-933.

[3] J.F. Bonder, J.D. Rossi and R. Ferreira, "Uniform bounds for the best Sobolev trace constant", Advanced Nonlinear Studies, 3 (2003), 181-192.

[4] H. Brezis, Functional Analysis, Sobolev Spaces and Partial Differential Equations, Springer, New York, (2011).

[5] E. DiBenedetto, Real Analysis, Birkhauser, Boston, (2001).

[6] L. C. Evans and R. F. Gariepy, Measure Theory and Fine Properties of Functions, CRC Press, Boca Raton (1992).

[7] T. Giorgi and R.G. Smits, "Principal eigenvalue estimates via the supremum of the torsion", Indiana U Math J. 59 (2010), 987-1011.

[8] P. Grisvard, Elliptic Problems in Non-smooth Domains, Pitman, Boston, (1985).

[9] G. Leoni, A First Course in Sobolev Spaces, American Math Society, Providence, (2009).

[10] F. Maggi, C. Vilani, "Balls have the worst best Sobolev inequalities", J. Geom. Anal. 15 (2005), $83-121$

[11] M. Motron, "Around the best constants for the Sobolev trace map from $W^{1,1}(\Omega)$ into $L^{1}(\partial \Omega, d \sigma)$ ", Asymptotic Analysis, 29 (2002), 69-90.

Department of Mathematics, University of Houston, Houston, Tx 77204-3008 USA

E-mail address: auchmuty@uh.edu 